\title{
Intervención cognitivo-conductual para la disminución del estrés en pacientes con cardiopatía isquémica
}

\section{Cognitive-behavioral Intervention to Reduce Stress in Patients with Ischemic Heart Disease}

\author{
Leonor Canales Reyes ${ }^{1}$ \\ Universidad Autónoma de Nueva León, México
}

(Rec: Noviembre 2010 - Acep: marzo 2011)

\begin{abstract}
Resumen
El objetivo de la presente investigación fue evaluar la eficacia de la intervención basada en la terapia cognitivo-conductual para disminuir el estrés percibido en pacientes con cardiopatía isquémica. El estudio corresponde a un diseño pre experimental, con evaluación pre y post intervención, con 9 participantes de edades entre los 40 y 60 años con cardiopatía isquémica. La intervención mediante el AIE (adiestramiento en inoculación al estrés), tiene una duración de 8 sesiones de dos horas cada una. Para medir el nivel de estrés antes y después se aplicó la Escala de Estrés Percibido. Se utilizó la prueba de rangos de Wilcoxon para evaluar los efectos de la intervención. Los resultados de la intervención demuestran que hubo una disminución significativa en los niveles de estrés al comparar el grupo antes y después de la intervención; asimismo, hubo una disminución en el nivel de estrés en cada uno de los participantes. Palabras clave: intervención cognitivo-conductual, estrés, cardiopatía isquémica.
\end{abstract}

\begin{abstract}
The aim of this paper was to evaluate the effectiveness of a cognitive-behavioral treatment to reduce the perceived stress level in patients with isquemic cardiopathy. This was a study with intervention without control group. Nine people with isquemic cardiophaty participated, their age were between 40 and 60 years old; all of them were patients of a public hospital in north of Mexico. Stress inoculation training was used in eight sessions' intervention; each session was about two hours. To measure stress level, Perceived Stress Scale was used, and Wilcoxon test to compare it before and after treatment. The main results confirmed a significant reduction of perceived stress after treatment; also, every participant had a decrease in the stress level.
\end{abstract}

Key words: cognitive-behavioral intervention, stress, ischemic heart disease.

\footnotetext{
1 Correspondencia a: Leonor Canales Reyes. Dirección Postal: Dr. Francisco L. Rocha 203, depto. 805, Colonia Residencial Galerías, CP 64640, Monterrey, Nuevo León, México. Tel: 01 (818) 33318 15. E-mail: leonorcanalesreyes@yahoo.com.mx.
} 


\section{Introducción}

Las intervenciones psicológicas han sido parte del tratamiento de personas con afecciones cardiacas en las últimas décadas. Los estudios publicados dan cuenta de los beneficios obtenidos para los pacientes. Entre las evidencias de esto se encuentra el Recurrent Coronary Prevention Project (RCPP) (Friedman et al., 1982) que ha demostrado que intervenciones centradas en la modificación de conducta resultan eficaces para reducir la morbilidad y mortalidad cardiaca. Los participantes de esta investigación (intervención en grupo) fueron 865 hombres y mujeres, víctimas de infarto al miocardio. Los resultados demostraron que la tasa de recurrencia de episodios cardiacos a los 60 meses fueron significativamente menores $(\mathrm{p}<0.005)$ para los pacientes del grupo experimental (12,9 por 100) que para los del control (21,2 por 100).

Por otro lado, el Lifestyle Heart Trial (LHT) (Ornish et al., 1990; Ornish., 1993) realizó un estudio con pacientes coronarios aleatoriamente asignados a un protocolo de tratamiento médico. Este constaba de ejercicio y dieta vegetariana estricta, además de seguir un programa de modificación del estilo de vida, manejo del estrés, a través de meditación y relajación. Los resultados mostraron un descenso en el nivel de colesterol $(20,5 \%)$ y los episodios de angina se redujeron a un $91 \%$. Se dedujo que es factible conseguir una regresión de la aterosclerosis coronaria mediante un programa de cambio de conducta y estilo de vida, en ausencia de tratamiento farmacológico.

En otro estudio, realizado por Burell (1996), 261 pacientes de ambos sexos sometidos a bypass coronario, participaron en un grupo control y un grupo de intervención conductual. El tratamiento constaba de la modificación del patrón de conducta tipo A, entrenamiento en solución de problemas, adiestramiento para hacer frente al dolor, ansiedad y estrés. La duración fue de un año, además de contar con sesiones de seguimiento durante el segundo y tercer año. Transcurridos entre cinco y seis años y medio desde la intervención quirúrgica, el grupo experimental presentó una tasa de mortalidad inferior a la del grupo control, además de mostrar una menor incidencia de episodios cardiovasculares como los infartos, nuevas intervenciones o angioplastías.

Para Fernández-Abascal, Martín y Domínguez (2003), la intervención conductual no se limita a trabajar solo factores de riesgo, sino que actúa junto al tratamiento, farmacológico, mejorando la adherencia al tratamiento; Asimismo, en lo que se refiere a la rehabilitación de un paciente que presente trastornos cardiovasculares, las técnicas conductuales han tenido como objetivo lograr el mantenimiento de las funciones cardiovasculares, además de instruir al paciente en habilidades de afrontamiento de estrés.

Respecto a la relación entre estrés y síntomas psicosomáticos, González y Landero (2006) exploraron la presencia o ausencia de síntomas psicosomáticos auto-reportados en estudiantes de psicología; la correlación entre los niveles de estrés y síntomas psicosomáticos entre hombres y mujeres y además se compararon los resultados entre los estudiantes de psicología de una universidad pública y una privada. Se trabajó con una muestra de 234 estudiantes de psicología, hallando presencia de síntomas psicosomáticos con una severidad media o alta en el 21,8\% de los estudiantes; una correlación significativa entre el estrés y síntomas psicosomáticos; mayor nivel de ambas variables en mujeres y diferencias significativas en síntomas psicosomáticos auto-reportados al comparar la universidad privada con la pública (González y Landero, 2006).

No solo el tratamiento cognitivo conductual en enfermedades cardiovasculares ha demostrado su eficacia, también se puede observar en intervenciones en VIH Sikkema (1998) describe un modelo de intervención cognitivo-conductual (Living Beyond Loss) para personas con VIH o familiares que han presentado la pérdida de un familiar, la pareja o un amigo por SIDA. Este modelo se basa en el desarrollo del apoyo social y cohesión del grupo, identificación y expresión de emociones relacionadas con el VIH y pérdidas, desarrollo de habilidades para el afrontamiento de dificultades al vivir con la infección por VIH y duelo del mismo, para reducir el estrés psicológico. En un estudio piloto se demostró que esta intervención reduce el estrés psicológico y las reacciones de duelo.

En síntesis, se estima que menos del 50\% de los programas de rehabilitación incluyen intervenciones psicológicas específicas (Rutledge, Linden, Davies y The Canadian Amlodipine/Atenolol in Silent Ischemia Study [CASIS] Investigators, 1999).

Como se puede observar, los programas de intervención cognitivo-conductual muestran resultados favorables y a pesar de que se han aplicado desde hace varios años, en algunos hospitales del Norte de México no se ofrecen como parte del tratamiento integral.

Con base en lo anterior y haciendo énfasis en la necesidad de integrar el tratamiento cognitivo conductual al tratamiento de los pacientes con 
cardiopatía isquémica, que actualmente no se ofrece en el hospital público en el que se trabajó, surge la necesidad de implementar un programa que se adecue a las condiciones del hospital y sus pacientes y que al mismo tiempo cumpla con los elementos de un tratamiento cognitivo-conductual, que permitan pronosticar su efectividad para el manejo del estrés.

Partiendo desde una perspectiva transaccional del estrés (Lazarus y Folkman, 1984), donde se define éste como una relación particular entre el individuo y el entorno que es evaluado por aquél como amenazante o desbordante de sus recursos y que pone en peligro su bienestar, se estableció como objetivo del estudio evaluar la eficacia de la intervención basada en la terapia cognitivo-conductual, para disminuir el estrés percibido en pacientes con cardiopatía isquémica.

Siguiendo con el concepto de estrés desde la perspectiva transaccional de Lazarus y Folkman, se buscó que mediante la intervención de tipo Cognitivo-Conductual, se modificara la valoración amenazante del estresor que incrementa los niveles de estrés. En esta investigación se valoró la eficacia en la reducción de estrés en los pacientes con diagnóstico de cardiopatía isquémica.

Es así que el propósito del presente estudio es evaluar si el tratamiento cognitivo conductual es efectivo en la disminución del estrés percibido en pacientes con cardiopatía isquémica.

Como se mencionó previamente, el hospital público no brinda atención psicológica como parte de la rehabilitación de los pacientes con cardiopatía isquémica. Además, los estudios citados demuestran que este tipo de intervenciones traen como consecuencia el control y manejo de la enfermedad, a través de intervenciones psicológicas que contribuyen a la mejoría del paciente. Al implementar la intervención basada en la terapia cognitivo-conductual y resolver los problemas que se presenten para completar las sesiones con la participación de los pacientes y el apoyo del centro médico, se podrá proponer al hospital, un plan factible para incorporar la rehabilitación psicológica como parte del tratamiento de los pacientes con cardiopatía isquémica. Así, esta atención traerá beneficios económicos, considerando que desde 1976 la Organización Mundial de la Salud reconoció los costos elevados del tratamiento de las enfermedades cardiovasculares, tanto desde el punto de vista humano como económico. (Comité de expertos de la OMS, 1993). En 1991, en Ginebra se llevó a cabo la reunión del Comité de Expertos sobre Rehabilitación de las Enfermedades Cardiovasculares, con especial atención a los países en desarrollo. El doctor HuChing-Li, subdirector general de la OMS, señaló que las enfermedades cardiovasculares constituyen una causa de mortalidad y morbilidad en países desarrollados (Comité de expertos de la OMS, 1993).

Asimismo, se requiere actualizar la rehabilitación en estos pacientes, incluyendo el aspecto psicológico. ya que en lo referente a los métodos de rehabilitación de los pacientes con enfermedades cardiovasculares, el Comité de Expertos en Rehabilitación de Pacientes con Enfermedades Cardiovasculares declaró un cambio en 1963; la rehabilitación con anterioridad consistía en la atención de personas en recuperación de un infarto agudo al miocardio sin complicaciones, para que los pacientes lograran mejores condiciones físicas, mentales y sociales posibles, con el fin de que pudieran, con sus propios esfuerzos, reasumir y mantener un lugar dentro de la comunidad (Organización Mundial de la Salud para Europa, 1969).

Finalmente, cabe mencionar que la Conferencia Sanitaria Panamericana reconoció en 2002 que en América Latina y en el Caribe, las enfermedades cardiovasculares son la primera causa de muerte prematura y de morbilidad (OPS, 2005). Razón por la cual, los trabajos realizados en este campo son de gran utilidad para la Salud Pública.

\section{Método}

\section{Participantes}

Se trabajó con un grupo experimental de 9 personas con cardiopatía isquémica pertenecientes al área de cardiología de un hospital público. Para decidir el tamaño del grupo experimental se tomó en cuenta que Sank y Shaffer (1993), al desarrollar un manual para la terapia cognitivo-conductual en grupos, recomiendan que el grupo se componga de 8 a 12 personas para un buen funcionamiento.

Se incluyeron en el estudio sujetos que presenten en su diagnóstico médico la enfermedad de cardiopatía isquémica, y que aceptaran participar en el estudio, comprometiéndose a asistir a todas las sesiones. Se excluyeron los pacientes próximos a una cirugía.

\section{Instrumentos}

Estrés percibido: se utilizó la Escala de Estrés Percibido -PSS- (Cohen, Kamarak y Mermelstein, 1983), en su versión para México de González y Landero (2007), que cuenta con adecuadas propiedades psicométricas. El coeficiente alfa de Cronbach 
reportado por los autores fue de .83 . El PSS consta de 14 reactivos con puntuación de nunca-0- a muy a menudo -4-, invirtiéndose la puntuación en los 7 ítems negativos. La mayor puntuación corresponde a mayor estrés percibido.

\section{Procedimiento}

Se invitó a las personas a ser participantes de una intervención cognitivo-conductual para la disminución de estrés. La intervención fue diseñada tomando en cuenta aspectos del servicio de atención al paciente con cardiopatía isquémica del hospital en que se trabajó, el número de sesiones de la intervención fue de 8 durante 5 semanas, la duración de cada sesión fue de aproximadamente 2 horas cada una; en dichas sesiones se trabajó con diferentes técnicas y temáticas como el estrés, su concepto, implicaciones médicas como las enfermedades, personalidades susceptibles al estrés y resistentes al mismo, la reestructuración cognitiva, inoculación al estrés, relajación, la comunicación, el estilo pasivo, agresivo y el asertivo, unidades subjetivas de ansiedad, autoestima y creatividad, enfocado al manejo del estrés. La intervención constó de 3 fases:

Primera fase: Conceptualización educativa, en la cual se comentó el efecto del estrés en el cuerpo humano. Se explicó la concepción transaccional del estrés y se establecieron las reglas del trabajo en grupo (confidencialidad, puntualidad, entre otros).

Segunda fase: Adquisición de habilidades a través de técnicas anteriormente escritas.

Tercera fase: Aplicación y consolidación del aprendizaje, fomentando la aplicación de las habilidades aprendidas a situaciones reales.

El grupo fue evaluado al inicio de la intervención y al término de la misma. Quienes se integraron en el estudio fueron invitados a participar de forma voluntaria, para lo cual se les pidió que firmaran un consentimiento informado. Se enfatizó la confidencialidad absoluta de los datos derivados de su situación clínica, omitiendo su nombre en el caso de que los resultados sean presentados en algún foro o publicación.
Para el análisis de los datos se utilizó una prueba no paramétrica debido al tamaño de muestra, usando la prueba de rangos de Wilcoxon para comparar los datos antes y después de la intervención.

\section{Resultados}

Los resultados que se presentan corresponden a 9 personas con cardiopatía isquémica (enfermedad incluyente), 3 hombres y 6 mujeres, todos casados, con edad y escolaridad promedio en el sistema educativo formal de 6,22 y 6,11 respectivamente. Solo dos personas tenían estudios superiores a primaria y ninguno había realizado estudios profesionales. Respecto a las enfermedades médicas diagnosticadas, 5 presentan solo infarto agudo al miocardio, 1 participante presentó problemas cardiacos y gastritis, 2 presentaron problemas cardiacos e hipertensión, y 1 participante presenta además de problemas cardiacos, diabetes e hipertensión. Seis de los nueve analizados practican ejercicio regularmente, con una media de 1,56 horas dedicadas a esta actividad a la semana. Respecto a la actividad laboral remunerada, solo 4 trabajan.

A continuación se describen los resultados correspondientes a cada uno de los objetivos del estudio.

\section{Resultados del diseño de la intervención}

La intervención diseñada fue adaptada a las condiciones del hospital (aulas, horarios, material, aparatos electrónicos, etc.) así como a los participantes (horarios, días, material, asistencia, etc.). Estas adaptaciones trajeron la adherencia total al programa, así como la satisfacción de los participantes.

Además de los notorios cambios y la disminución del estrés (a través de las escalas), sus comentarios corroboran la satisfacción al haber participado en la intervención: "Uno de los aprendizajes del curso fue la relajación, la cual debe ser una práctica diaria cuando me siento ansioso o estresado"; "debemos de aprender a controlar esta ansiedad, a manejar mis

Tabla 1. Comparación de estrés antes y después de la intervención

\begin{tabular}{lcc}
\hline \multicolumn{1}{c}{ Síntomas } & Antes & Después \\
\hline Mediana & 25.00 & 16.00 \\
Media & 27.88 & 15.22 \\
Desviación estándar & 8.92 & 4.14 \\
Prueba de rangos de Wilcoxon & & $\mathrm{Z}=-2.666 ; \mathrm{p}=.008$ \\
\hline
\end{tabular}


Figura 1. Gráfica de estrés por participante

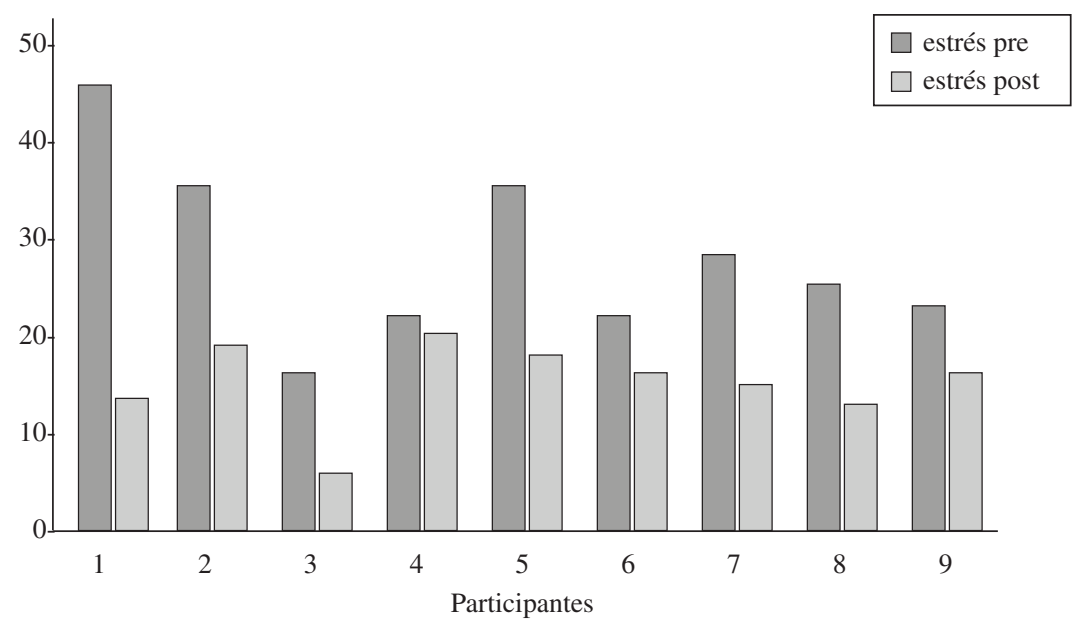

corajes"; " noté cambios desde el primer día al poder hacer ejercicios en mi casa"; "me sentía angustiado"; "no podía conciliar el sueño"; "me sentía en un pozo en el que estaba yo metido"; "no encontraba yo solución al problema"; "me sentía desesperado"; "el hecho de que no pudiera hacer una vida como la demás gente"; "encontré la respuesta al problema por el que estábamos pasando"; "he sentido un cambio en mi persona, en el modo de reaccionar, pues he dominado esa ansiedad por medio de los ejercicios que he estado llevando a cabo"; "me siento contento y agradecido"; "nos proporcionó una enseñanza muy valiosa"; "no soportaba mi cabeza ni mi quijada, además de tener insomnio"; "con las técnicas aprendidas he logrado mejorarme y estar más tranquila".

\section{Eficacia de la intervención para la disminución del estrés}

El objetivo principal de este trabajo fue diseñar una intervención basada en la terapia cognitivoconductual, adaptada a las condiciones del hospital donde se instrumentó el estudio y a las características particulares de sus usuarios. Además de evaluar la eficacia de la misma, se puede observar el logro de éste, ya que el puntaje máximo de la escala es de 56, y respecto al máximo posible, los participantes estaban antes de la intervención en casi un 50\% de esta totalidad (en donde el 56 representa un 100\%), esto es reflejado tanto en la media como en la mediana. Al ser la media más alta que la mediana, se puede observar que hay personas con puntajes más altos que hacen que el promedio de estrés sea mayor, la desviación estándar indica que hay variabilidad en los puntajes. Después de la intervención, se observa una disminución considerable, siendo la media menor que la mediana y la desviación estándar menor que al inicio, es decir, los puntajes son más bajos con una variabilidad menor.

La variable de interés para la intervención fue el estrés, cuya media antes de la intervención fue de $27.9(\mathrm{DE}=8.9)$ y después de la intervención, de 15.2 ( $\mathrm{DE}=4.2$ ), encontrándose una diferencia significativa $(\mathrm{p}=0.008)$ con la prueba de rangos de Wilcoxon (Tabla 1).

El participante 1 fue el que más disminuyó su estrés en comparación con el estrés percibido al inicio y al final con los otros estudiados; el número 3 fue el de más baja percepción de estrés al inicio, pero fue, además, el más bajo al final de la intervención. Además, se observó que todos disminuyeron su estrés.

$\mathrm{Al}$ realizar la comparación antes y después del curso, de las variables de interés, se registró un decremento significativo del estrés $(Z=-2.666 ; p$ $=.008)$. Este resultado comprueba la eficacia de la intervención para la disminución del estrés.

Al comparar cada ítem de la escala de estrés, se observa una disminución en las media en todos los casos, excepto en el ítem 10, que no presenta cambios. En la tabla 2 se señalan los cambios significativos.

\section{Discusión y conclusiones}

El presente capítulo tiene como propósito analizar las implicaciones de los resultados obtenidos para la Psicocardiología, resaltando los puntos centrales del trabajo de investigación. En capítulo se 
Tabla 2. Comparación de ítems de la escala de estrés antes y después de la intervención.

\begin{tabular}{|c|c|c|c|c|c|c|c|c|}
\hline & \multicolumn{2}{|c|}{ Media } & \multicolumn{2}{|c|}{$\begin{array}{c}\text { Desviación } \\
\text { estándar }\end{array}$} & \multicolumn{2}{|c|}{$\begin{array}{l}\text { Rangos } \\
\text { medios }\end{array}$} & \multicolumn{2}{|c|}{ Wilcoxon } \\
\hline & Pre & post & Pre & post & + & - & Z & $\mathrm{p}$ \\
\hline $\begin{array}{l}\text { E1. ¿Con qué frecuencia has estado afectado por } \\
\text { algo que ha ocurrido inesperadamente? }\end{array}$ & 2.4 & 1.9 & 1.2 & 1.1 & 3.00 & 4.40 & -1.406 & .160 \\
\hline $\begin{array}{l}\text { E2. ¿Con qué frecuencia te has sentido incapaz de } \\
\text { controlar las cosas importantes de tu vida? }\end{array}$ & 2.2 & 0.9 & 1.3 & 0.8 & .00 & 3.50 & -2.226 & .026 \\
\hline $\begin{array}{l}\text { E3. ¿Con qué frecuencia te has sentido nervioso } \\
\text { o estresado (lleno de tensión)? }\end{array}$ & 3.2 & 1.7 & 0.8 & 0.5 & .00 & 4.50 & -2.565 & .010 \\
\hline $\begin{array}{l}\text { E4. ¿Con qué frecuencia has manejado con éxito } \\
\text { los pequeños problemas irritantes de la vida? }\end{array}$ & 1.3 & 0.4 & 0.9 & 0.7 & .00 & 3.50 & -2.271 & .023 \\
\hline $\begin{array}{l}\text { E5. ¿Con qué frecuencia has sentido que has } \\
\text { afrontado efectivamente los cambios importantes } \\
\text { que han estado ocurriendo en tu vida? }\end{array}$ & 1.0 & 0.2 & 0.7 & 0.4 & .00 & 3.50 & -2.333 & .020 \\
\hline $\begin{array}{l}\text { E6. ¿Con qué frecuencia has estado seguro sobre } \\
\text { tu capacidad de manejar tus problemas personales? }\end{array}$ & 2.1 & 1.0 & 1.5 & 0.5 & 2.00 & 4.33 & -2.058 & .040 \\
\hline $\begin{array}{l}\text { E7. ¿Con qué frecuencia has sentido que las cosas } \\
\text { te van bien? }\end{array}$ & 1.7 & 0.8 & 1.1 & 0.7 & 3.00 & 4.17 & -1.930 & .054 \\
\hline $\begin{array}{l}\text { E8. ¿Con qué frecuencia has sentido que no podías } \\
\text { afrontar todas las cosas que tenías que hacer? }\end{array}$ & 2.2 & 1.4 & 1.6 & 1.2 & 4.67 & 5.17 & -1.021 & .307 \\
\hline $\begin{array}{l}\text { E9. ¿Con qué frecuencia has podido controlar las } \\
\text { dificultades de tu vida? }\end{array}$ & 1.4 & 0.8 & 0.9 & 0.7 & .00 & 2.00 & -1.604 & .109 \\
\hline $\begin{array}{l}\text { E10. ¿Con qué frecuencia has sentido que tienes } \\
\text { el control de todo? }\end{array}$ & 1.7 & 1.7 & 1.4 & 0.9 & 4.33 & 3.75 & -.175 & .861 \\
\hline $\begin{array}{l}\text { E11. ¿Con qué frecuencia has estado enfadado } \\
\text { porque las cosas que te han ocurrido estaban fuera } \\
\text { de tu control? }\end{array}$ & 2.2 & 1.0 & 1.1 & 0.5 & 2.50 & 4.79 & -2.209 & .027 \\
\hline $\begin{array}{l}\text { E12. ¿Con qué frecuencia has pensado sobre las } \\
\text { cosas que no has terminado (pendientes de hacer)? }\end{array}$ & 2.9 & 1.3 & 0.9 & 0.9 & .00 & 4.00 & -2.392 & .017 \\
\hline $\begin{array}{l}\text { E13. ¿Con qué frecuencia has podido controlar la } \\
\text { forma de pasar el tiempo (organizar)? }\end{array}$ & 1.3 & 0.9 & 0.9 & 0.9 & 2.50 & 3.13 & -1.414 & .157 \\
\hline $\begin{array}{l}\text { E 14. ¿Con qué frecuencia has sentido que las } \\
\text { dificultades se acumulan tanto que no puedes } \\
\text { superarlas? }\end{array}$ & 2.1 & 1.2 & 1.2 & 1.2 & .00 & 3.00 & -2.060 & .039 \\
\hline
\end{tabular}

sigue la secuencia utilizada en apartados anteriores para facilitar su comprensión.

Para el diseño de la intervención se tomó como base el trabajo realizado por González con personas con VIH (2002), sobre inoculación al estrés, a través del cual se trabajó una primera fase, Conceptualización o educativa, en el que los puntos se centraban en comentar el efecto del estrés en el cuerpo humano, explicar la concepción transaccional del estrés, establecer las reglas del trabajo en grupo (confidencialidad, puntualidad, entre otros). En la presente investigación se diseñó, además, un apoyo visual que se presentó en cada una de la sesiones para darle seguimiento a cada actividad dentro de ellas, y que el trabajo realizado cumpliera con el objetivo de las sesiones. Debido a que la muestra presentó una media de 6.1 años en el sistema educativo formal, el apoyo mencionado brindó guía y facilitó la comprensión de las temáticas abordadas en esta fase de la intervención.

En la segunda fase, adquisición de habilidades, se utilizaron técnicas de relajación, reestructuración cognitiva, entrenamiento asertivo, solución de problemas.

La relajación es la técnica recomendada por Meichenbaum (1988) para iniciar cualquier tratamiento de manejo del estrés. La reestructuración cognitiva es una técnica utilizada en la terapia cognitivo conductual para modificar la valoración e interpretación subjetiva. Modificar la percepción de las situaciones de estrés es la base de una intervención enmarcada en la teoría transaccional de Lazarus y Folkman (1984); debido a que el instrumento utilizado para medir la eficacia de la intervención evaluó estrés percibido, esta técnica fue indispensable en el proceso. El entrenamiento asertivo es necesario para 
el trabajo con pacientes con cardiopatía isquémica por la personalidad tipo A que los caracteriza; la personalidad tipo A (PCTA) fue desarrollada por Rosenman y Friedman (1959), quienes explicaron un conjunto de características presentes en pacientes coronarios, entre las que destacaban: un esfuerzo intenso y mantenido hacia el logro de objetivos, elevada competitividad, baja tolerancia a la frustración, búsqueda de reconocimiento, impaciencia, multitasking, hostilidad, entre otras. A su vez, la solución de problemas es una técnica que desarrolla nuevos repertorios de conducta ante diferentes situaciones o problemas (D' Zurilla y Goldfried, 1971). Al ser utilizada en la intervención, los ejemplos trabajados fueron referentes a problemáticas de su enfermedad.

La tercera fase, aplicación y consolidación del aprendizaje, fomentó la aplicación de las habilidades aprendidas a situaciones reales, con el propósito de que las habilidades adquiridas fueran utilizadas en la vida cotidiana y no quedaran en información teórica, como se comentó previamente en el capítulo de resultados, donde los participantes hablaron sobre el impacto de la intervención en su vida cotidiana.

El objetivo principal de la investigación fue evaluar la eficacia de la intervención cognitivoconductual. Los resultados muestran una disminución de la percepción de estrés, donde los puntajes iniciales fueron de 27.88 y después de 15.22 , evidenciando una disminución significativa al utilizar la prueba estadística de rangos de Wilcoxon, además de encontrarse una disminución en cada uno de los participantes. Sin embargo, al analizar los datos de cada ítem no existe una disminución significativa en todos los casos, lo que puede explicarse por el tamaño de la muestra. No obstante, la media es menor después de la intervención en todos los ítems, a excepción del ítem 10, que hace referencia al control en la vida de la persona. Los pacientes con enfermedades crónicas, en ocasiones, pueden percibir la falta de control en los aspectos de su vida.

Comparando el puntaje inicial obtenido, podemos concluir que los participantes en la intervención tenían un alto nivel de estrés, pues en diferentes muestras, los puntajes de la misma escala PSS son menores; en estudiantes de licenciatura, la media fue de 21.9 (González y Landero, 2008); en personas con VIH, la media fue de 22.6 (Remor, 2006); en pacientes con epidermolisis bullosa, la media obtenida fue de 21.7 (Reyna, 2009); incluso en una muestra de pacientes con el mismo diagnóstico que los partipantes de este estudio, la media de estrés fue de 24.7 (García, 2009). Al finalizar la intervención, el puntaje de estrés percibido fue menor en comparación con cada una de las muestras mencionadas, lo cual permite atribuir los resultados de este estudio al uso de una intervención cognitivo conductual en enfermedades crónicas.

La terapia cognitivo conductual ha demostrado su efectividad a lo largo de los años, como podemos observarlo en los estudios realizados por diferentes autores en el uso de estrategias cognitivo-conductuales. Por ejemplo, el metaanálisis realizado por Rossy y colaboradores (1999) con 49 estudios, a partir del que se concluye que el tratamiento multidisciplinar debe incluir la terapia cognitivo-conductual, y el estudio de Moioli y Merayo (2005), reportando que valores de ansiedad, dolor y depresión, al ser manejados en la intervención cognitivo-conductual en personas con fibromialgia, reportan diferencias significativas y un incremento de actividades en la vida diaria.

La cantidad de evidencia en favor del uso de tratamientos cognitivo conductuales respalda la recomendación de incluirlo para pacientes con cardiopatía isquémica que la OMS señala que deben de brindar en los servicios de cardiología, aun cuando existen limitaciones del presente estudio relacionadas al tamaño y selección de la muestra, además de no haber contado con grupo control que sirviera de comparación con el grupo experimental por la poca disponibilidad de los participantes, a pesar de la difusión por radio, televisión y medios impresos, donde fue expresada la convocatoria para formar parte de esta investigación, que, además de gratuita, fue otorgada junto a material de apoyo denominado "cuaderno de trabajo" para que los participantes recordaran las actividades y técnicas aprendidas.

La cardiopatía isquémica forma parte de un sinnúmero de enfermedades denominadas "crónicas", las cuales se caracterizan por ser de larga duración, cuyo fin no puede preverse claramente. Para Velasco y Sinibaldi (2001), estas enfermedades presentan fases en su historia natural, las cuales son: fase de crisis de iniciación, fase crónica y la fase terminal. En la fase crónica, los pacientes deben de aprender a vivir día a día con la enfermedad; además tanto el paciente como la familia presentan poca habilidad para llevar a cabo una vida normal; existen creencias de recuperación de normalidad cuando el paciente se "cure", por lo que en muchas ocasiones pueden presentarse pensamientos de vergüenza y/o culpabilidad por no lograrlo, entre otras características; las mismas que presentaban cada uno de los participantes de la investigación. Es importante conocer las etapas por las que atraviesa cada paciente y más si hablamos de 
terapias grupales, donde se deben tomar en cuenta los avances o retrocesos de cada uno para que no interfiera con la intervención.

Las aportaciones de esta investigación en referencia a la educación: se sugiere que se trabaje como un curso, no como terapia grupal, que se cuente con una guía (manual) a través del apoyo que puede brindar el paquete de Microsoft office de Power point, ya que ayuda a la adherencia al tratamiento y evita el rechazo que en ocasiones se presenta por la estigmatización del psicólogo en México.

\section{Referencias}

Burell, G. (1996). Behavioral medicine interventions in secondary prevention of coronary Herat disease. En K. Orth-Gomér y N. Schneiderman (Eds.), Behavioral Medicine Approaches to Cardiovascular Disease Prevention (227-236). Mahwah: Lawrence Erlbaum.

Cohen, S., Kamarak, T. \& Mermelstein, R. (1983). A Global Measure of Perceived Stress. Journal of health and social behavior, 24, 385-396.

Comité de Expertos de la OMS (1993). Rehabilitación después de las Enfermedades Cardiovasculares, con especial atención a los países en desarrollo. Informe de Ginebra, Organización Mundial de la Salud, 1993. Serie de Informes Técnicos, $\mathrm{N}^{\circ} 831$.

Fernández-Abascal, E. G., Martín, M. D. \& Domínguez, F. J. (2003). Factores de riesgo e intervenciones psicológicas eficaces en los trastornos cardiovasculares. Psicohema, 15(4), 615-630.

Friedman, M., Thorensen, C. E., Gill, J. J., Ulmer, D., Thompson, L., Powell, L., Prince, V., Eleck, S. R., Rabin, D., Breall, W. S., Piaget, G., Dixon, T., Bourg, E., Levy, R. \& Tasto, D. (1982). Recurrent coronary prevention project study: Methods, baseline results and preliminary findings. Circulation, 66, 83-92.

García, R. A. (2008). Estrés, afrontamiento y optimismo en pacientes con cardiopatía isquémica. Tesis de maestría. Facultad de Psicología, U.A.N.L. (Diciembre 2008).

González, M.T. (2002). Intervención cognitivo-conductual para el manejo del estrés en personas que viven con VIH/ SIDA. Tesis de maestría. Facultad de Psicología, U.A.N.L. (Febrero 2002). Disponible en: http://cdigital.dgb.uanl.mx/ te/1080124350/1080124350.html

González, M. T. \& Landero, R. (2006). Síntomas psicosomáticos auto-informados y estrés en estudiantes de psicología. Psicología Social, 21 (2), 141-152.
González, M. T. \& Landero, R. (2007). Factor Structure of the Perceived Stress Scale (PSS) in a Sample from Mexico. The Spanish Journal of Psychology, 10 (1), 199-206.

González, M. T. \& Landero, R. (2008). Confirmación de un modelo explicativo del estrés y de los síntomas psicosomáticos mediante ecuaciones estructurales. Revista Panamericana de Salud Pública, 23 (1), 7-18.

Lazarus, R. \& Folkman, S. (1984). Stress, coping and adaptation. New York: Springer.

Meichenbaum, D. (1988). Manual de Inoculación de Estrés. México: Editorial Martínez Roca.

Ornish, D., Brown, S. E., Scherwitz, L. S., Billings, J. H., Amstrong, W. T., Ports, T. A., McLanaham, S. M., Kirkeeide, R. L., Brand, R. J. \& Gould, K. L. (1990). Can lifestyle change reverse coronary heart disease? Lancet, 336, 129-133.

Ornish, D., Scherwitz, L. S., Doody, R. S., Kerten, D., McLanaham, S. M., Brown, S. E., DePuey, G., Sonnemaker, R., Haynes, C., Lester, J., McAlister, G. K., Hall, R. J., Burdine, J. A. \& Gotto, A. M. (1993). Effects of stress management training and dietary changes in treating ischemic heart disease. Journal of American Medical Association, 249, 54-60.

OPS (2005, septiembre 23). Enfermedades cardiovasculares: más allá del corazón. Comunicado de Prensa Organización Panamericana de la Salud. Consultado el 24 de abril de 2007. Disponible en: http://www.paho.org/Spanish/DD/PIN/ ps050923.htm

Remor, E. \& Carrobles, J. (2001). Versión Española de la escala de estrés percibido (PSS-14): Estudio psicométrico en una muestra VIH+. Ansiedad y Estrés, 7 (2-3), 195-201.

Reyna, J. A. \& González, M. T. (2008). Estrés y calidad de vida en personas con Epidermólisis Bullosa. Artículo enviado para publicación.

Rutledge, T., Linden, W., Davies, R. F. \& The Canadian Amlodipine/Atenolol in Silent Ischemia Study (CASIS) Investigators (1999). Psychological risk factors may moderate pharmacological treatment effects among ischemic heart disease patients. Psychosomatic Medicine, 61, 834-841.

Sank, L. I. \& Shaffer, C. S. (1993). Manual del terapeuta para la terapia Cognitiva Conductual en grupos. España: Editorial Desclée de Brouwer.

Sikkema, K. J. (1998). Public interest directorate: Living Beyond Loss: An intervention for Coping with HIV and HIV Related Loss. American Psychological Association. Revisado el 4 de Junio de 2007. Disponible en: http://www.apa.org/pi/aids/ sikkema.html.

Velasco, L. \& Sinibaldi, J. (2001). Manejo del Enfermo Crónico y su familia. México: Manual Moderno. 